\title{
Development of a CFD Analysis Plan for the First VHTR Standard Problem
}

\section{$4^{\text {th }}$ International Topical Meeting on High Temperature Reactor Technology}

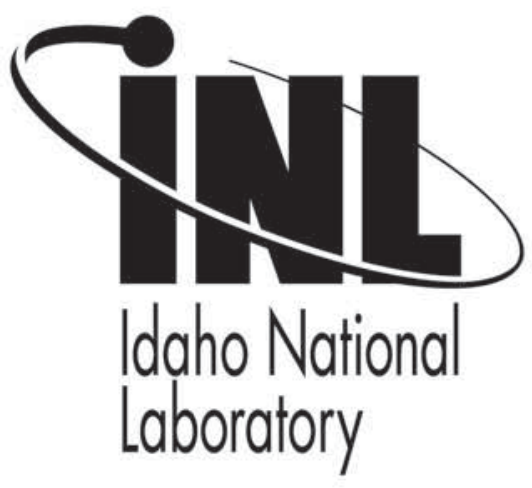

\author{
Richard W. Johnson
}

September 2008

This is a preprint of a paper intended for publication in a journal or proceedings. Since changes may be made before publication, this preprint should not be cited or reproduced without permission of the author. This document was prepared as an account of work sponsored by an agency of the United States Government. Neither the United States Government nor any agency thereof, or any of their employees, makes any warranty, expressed or implied, or assumes any legal liability or responsibility for any third party's use, or the results of such use, of any information, apparatus, product or process disclosed in this report, or represents that its use by such third party would not infringe privately owned rights. The views expressed in this paper are not necessarily those of the United States Government or the sponsoring agency. 
HTR2008-58283

\title{
DEVELOPMENT OF A CFD ANALYSIS PLAN FOR THE FIRST VHTR STANDARD PROBLEM
}

\author{
Richard W. Johnson \\ Idaho National Laboratory \\ Idaho Falls, ID 83415, USA
}

\begin{abstract}
Data from a scaled model of a portion of the lower plenum of the helium-cooled very high temperature reactor (VHTR) are under consideration for acceptance as a computational fluid dynamics (CFD) validation data set or standard problem. A CFD analysis will help determine if the scaled model is a suitable geometry for validation data. The present article describes the development of an analysis plan for the CFD model. The plan examines the boundary conditions that should be used, the extent of the computational domain that should be included and which turbulence models need not be examined against the data. Calculations are made for a closely related 2D geometry to address these issues. It was found that a CFD model that includes only the inside of the scaled model in its computational domain is adequate for CFD calculations. The realizable $\mathrm{k} \sim \mathcal{E}$ model was found not to be suitable for this problem because it did not predict vortex-shedding.
\end{abstract}

Keywords: CFD; VHTR; NGNP; analysis plan; turbulence model, validation

\section{INTRODUCTION}

The reference design for the prismatic Next Generation Nuclear Power Plant (NGNP) is based on the General Atomics very-high temperature reactor (VHTR) concept which in turn is based on their Gas Turbine - Modular Helium Reactor (GTMHR) [1]. A major concern in the operation of this design is the nature of the flow of hot gas as it exits the core into the lower plenum and then out the vessel exit duct. Two main concerns are the impingement of hot jets onto the walls of the lower plenum and the degree of mixing of the helium coolant with spatially varying temperature as it flows out of the reactor vessel. Computational fluid dynamic (CFD) simulations are proposed to obtain the needed local fluid dynamic and temperature information.

Successful CFD simulations require careful planning and extensive calculations to help ensure that they accurately represent the flow physics. An important aspect of the application of CFD is to validate the results against detailed validation data, often for scaled problems that involve similar physics. A scaled section of the lower plenum of the reference prismatic NGNP was designed at the Idaho National Laboratory; see Figure 1. Extensive flow data have been taken in the scaled model while installed in the INL's matched index of refraction (MIR) test facility [2]. The MIR data are under consideration to become a validation data set or Standard Problem for CFD-based safety analysis of the VHTR. A CFD analysis to simulate the flow data from the MIR is being undertaken to determine if the flow geometry is suitable for a validation data set. The present article reports the development of an analysis plan for the CFD analysis.

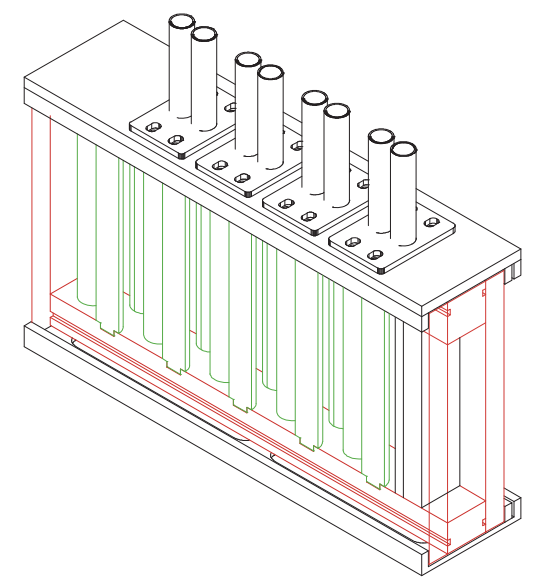

Fig 1. Drawing of the scaled model used in the INL MIR facility to obtain flow data. Flow enters in from the top inlets and flows out toward the left rear; half and full cylinders represent core support columns.

An important aspect of the analysis plan is to determine the extent of the computational domain necessary such that the boundary conditions employed are suitable for the problem computed. The scaled model was installed in the MIR such 
that the flow medium (mineral oil) flowed both inside and around the outside of the model. It is necessary to know if the outer flow will need to be modeled along with the inner flow for the present model geometry in order to produce accurate results. That is, is there a suitable outflow boundary condition that can be used for a CFD model that incorporates only the flow inside the model that will allow proper comparisons between calculations and the experimental data?

The scaled model involves flow around several interior posts representing the core block support columns in the lower plenum of the reference prismatic NGNP design. It is expected that the flow will therefore involve shedding vortices at multiple locations as well as turbulence, making the flow nonstationary. It is further desirable to determine which turbulence models are not appropriate for simulating the flow. In fact, it has been found that some two-equation turbulence models do not allow vortex-shedding, probably because of excessive diffusion. Numerical practices employed are based on the ASME Journal of Fluids Engineering 'Statement of Numerical Accuracy.'

\section{ANALYSIS PLAN}

The overall objective of the analysis plan is to determine the best approach for the numerical analysis that will yield results that most closely represent the physics of the actual flow. This includes a determination of the extent of the computational domain, the most appropriate boundary conditions to use, the fineness of the grid to achieve grid convergence, the appropriate tolerance for iterative convergence at each time step and which turbulence models can be used to compare to the experimental data.

The first requirement to be applied to the analysis plan is that the CFD code to be used be one that is fully operational and currently and easily available to all interested parties - the National Laboratories, the reactor vendors and the U.S. Nuclear Regulatory Commission (NRC), that is, a commercial CFD code. The code used initially for the development of the analysis plan is FLUENT [3].

It was deemed appropriate to use a 2D CFD model of the 3D scaled model to develop the analysis plan because the geometry of the actual 3D scaled model is invariant in the third dimension below the plane of the inlet jets and should provide a good idea of how domain considerations and boundary conditions will apply in 3D. Also, 2D calculations require much less time than $3 \mathrm{D}$ calculations.

Initial calculations were made using a flow domain that included only the flow region inside the scaled model. Figure 2 illustrates the cross-section of the scaled model. Flow is from right to left in the scaled model as shown. The final 2D CFD model was constructed to include some, but not all, of the full and half posts in the scaled model. The last four full posts and the last three pairs of half cylinders were included. Figure 3 illustrates the 2D CFD model employed for the inner domain CFD model.

\section{Top View}

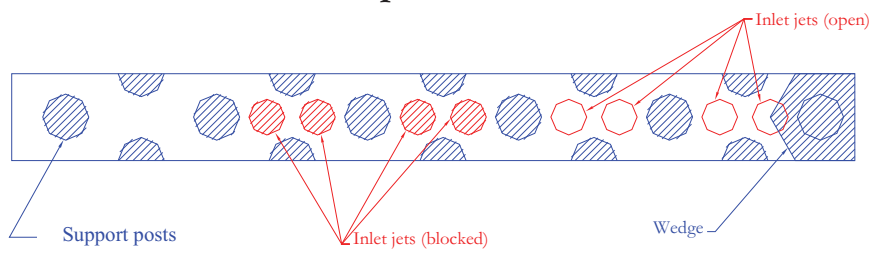

Figure 2. Top view of the scaled model showing full and half support posts and inlet jets (ports).

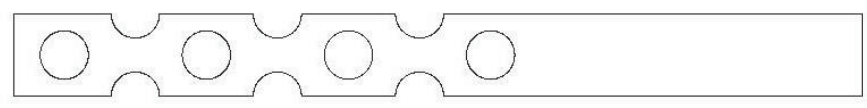

Figure 3. 2D CFD model for flow inside the scaled model.

Initial 2D calculations were performed for grid convergence studies with a model similar to that shown in Fig. 3 , except that only the last two full posts and last two pairs of half posts were included. The realizable two-equation $\mathrm{k} \sim \varepsilon$ model employing the enhanced wall treatment was used; the $\mathrm{y}^{+}$distribution of the near-wall cell ranged between 0.1 and 6 , appropriate to the use of the low Reynolds number wall model approach of the enhanced wall treatment. No vortex shedding could be obtained, even when substantial numerical perturbations were applied to the inlet velocity profile. No vortex shedding was obtained when the wall treatment was changed to standard wall functions (using the same grid); standard wall functions are constructed to be applicable to use in the region very close to the wall [3].

Vortex shedding was obtained in an earlier numerical study of flow in a tube bundle by the author [4] using the Reynolds stress model (RSM) wherein a separate transport equation is solved for each Reynolds stress. When the RSM was employed in the present study, clear vortex-shedding was obtained, even without any numerical kick applied. The RSM was used throughout the rest of the calculations made in the development of the analysis plan reported herein. While it is not appropriate to determine which turbulence model is most appropriate to simulate the 3D flow physics until 3D calculations are made, it appears justified to eliminate the realizable $\mathrm{k} \sim \mathcal{\varepsilon}$ model from contention inasmuch as it apparently disallows vortex-shedding, probably due to the excessive turbulent diffusion of momentum.

The two clear candidates for outlet boundary condition in FLUENT are the "outflow" and the "pressure-outlet" conditions. The former enforces a constant gradient condition to the velocity components while the latter sets a constant static pressure condition just downstream of the outlet plane. Actually, neither of these conditions is correct because the outlet to the inner flow domain is just barely downstream of the last full post such that there is vortex shedding occurring at the outlet plane. It is expected that using just the inner flow for the computational domain is inappropriate; however, it may be the case that numerical simulations for the case of the inner 
domain only will match simulations for the case of including the outer domain for some region within the inner domain.

Simulations were made using the second-order in space convective-differencing scheme QUICK with second-order implicit time stepping available in FLUENT [3]; the secondorder implicit time scheme requires a constant time step. The RSM turbulence model was used with standard wall functions; ' $\mathrm{y}^{+}$' values ranged from 0.1 to 6 . Three grids $(\mathrm{a}, \mathrm{b}, \mathrm{c})$ were used to investigate grid independence. The grids had 27,232; 67,171 ; and 113,046 cells. Factors of 1.5 and 1.35 were used on mesh face edges to refine the successive grids. Regions of structured cells were constructed around the posts and halfposts and in the region upstream of the posts. Other regions were paved. Figure 4 provides a close-up of the intermediate grid illustrating its construction.

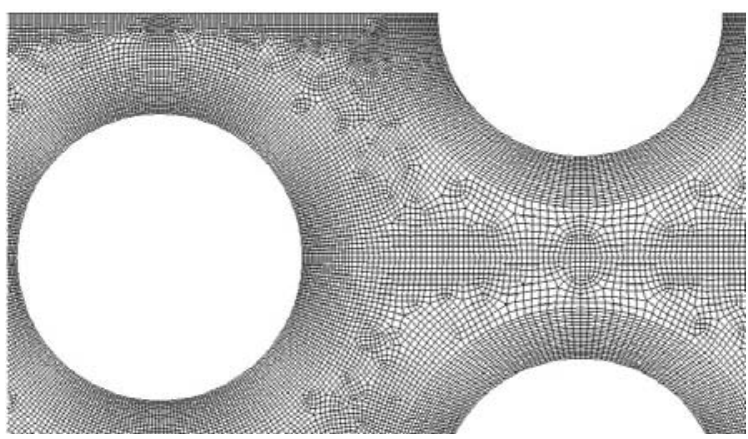

Figure 4. Mesh detail of intermediate grid.

The 'pressure-outlet' condition was used at the outlets of the 'inside-only' CFD models for the grid study. 'Scaled' tolerances [3] of approximately $1 \times 10^{-6}$ for the velocities and continuity and $1 \times 10^{-5}$ for turbulence quantities were obtained for the inner iterations. These tolerances were found to be adequate in a related study [4]. A time step of $3.5 \times 10^{-4} \mathrm{sec}$ was used for the coarsest grid; time steps of $1.0 \times 10^{-4} \mathrm{sec}$ were used for the two finer grids. Figure 5 shows a time trace for grid ' $b$ ' for the Reynolds-averaged streamwise velocity ' $u$ ', which is unsteady due to vortex-shedding, with a symbol at every $5^{\text {th }}$ time step. The point lies just behind a full post. The time step is fine enough to capture the unsteadiness in the solution. Figure 5 indicates multiple time scales. These are likely due to multiple vortex-shedding sites.

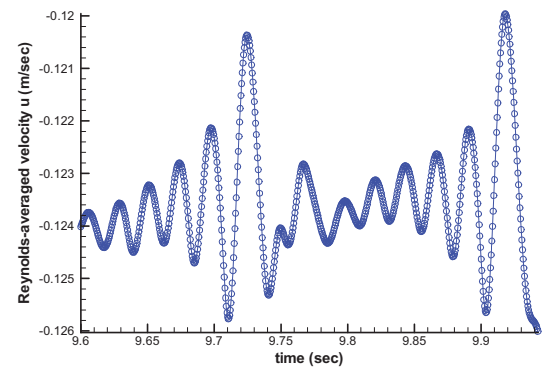

Figure 5. Time trace of the Reynolds-averaged streamwise velocity ' $u$ ' for the intermediate grid.

To be able to compare outlet flow conditions between cases, it is necessary to look at the long-time average of the flow. The experimental data were taken using a two-camera PIV system. There were 750 data pairs taken (from which the velocities were computed) over typical time intervals of 375 seconds, representing very long-time averages of the flow variables [5]. The question is: Over how long of a time period must the unsteady computations be made to obtain a long-time average with an acceptable error? FLUENT provides for the long time average of the Reynolds-averaged flow quantities.

Figures 6-8 plot the normalized $l_{2}$ norm of the long-time averaged streamwise (U) and transverse (V) velocity profiles at the outlet to the physical model with time for grids $\mathrm{a}, \mathrm{b}$ and c. The normalized $l_{2}$ norm is computed based on:

$$
l_{2}=\left[\sum_{i=1}^{N}\left(s-s_{o}\right)^{2}\right]^{1 / 2} /\left[\sum_{i=1}^{N} s_{o}^{2}\right]^{1 / 2}
$$

where $s$ is the quantity in question, $s_{o}$ is a reference value (based on the last profile computed) and $N$ is the number of nodes in a profile. The difference should either be low or not changing very much.

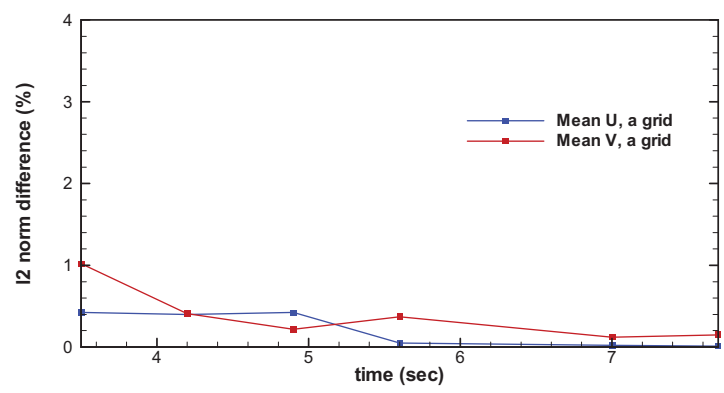

Figure 6. $l_{2}$ normalized differences for grid 'a' for $\mathrm{U}$ and $\mathrm{V}$. Ref. time $=8.575 \mathrm{sec}$.

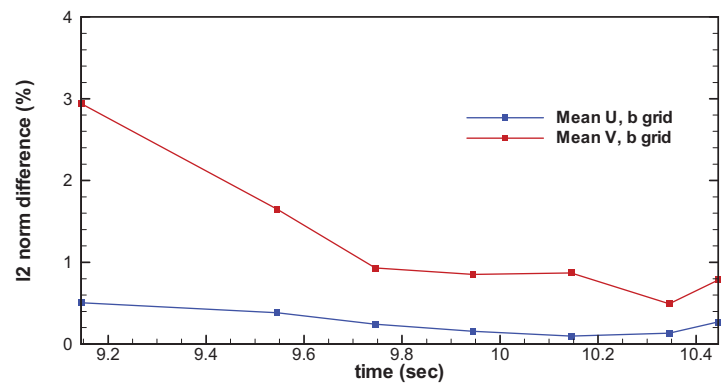

Figure 7. $l_{2}$ normalized differences for grid ' $b$ ' for $\mathrm{U}$ and $\mathrm{V}$. Ref. time $=10.5451 \mathrm{sec}$.

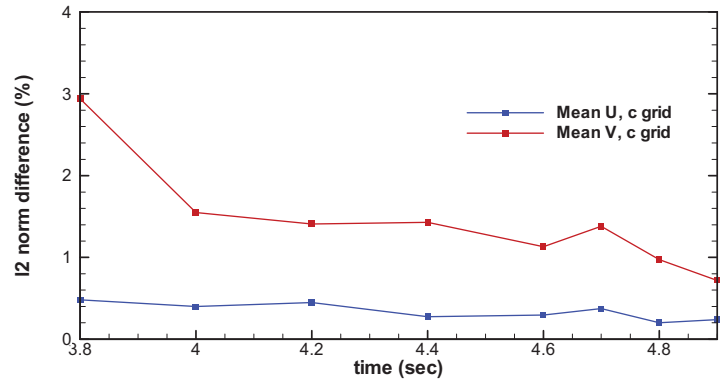

Figure $8 . l_{2}$ normalized differences for grid ' $\mathrm{c}$ ' for $\mathrm{U}$ and $\mathrm{V}$. Ref. time $=5.0 \mathrm{sec}$. 
Figures 9 and 10 illustrate the long-time averaged or mean velocity profiles for $\mathrm{U}$ and $\mathrm{V}$ at the CFD model outlet for the three grids. The results for the coarsest grid, ' $a$ ', are visibly different from the other results. The mean $\mathrm{U}$ results for the ' $\mathrm{b}$ ' and ' $\mathrm{c}$ ' grids appear very close. The results for mean $\mathrm{V}$ indicate that results on the finest two grids, ' $b$ ' and ' $c$ ', are not quite symmetric. The variations due to asymmetry are greater than the variations due to grid refinement. One can take the view that there may be some effect that has a relatively long time scale that has not yet been captured in the time scales of the present simulations, but that grid ' $b$ ' is provisionally appropriate for further calculations.

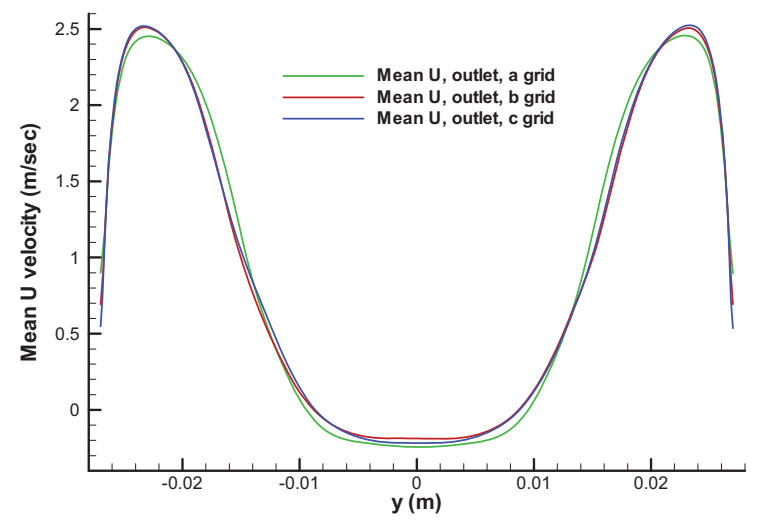

Figure 9. Comparison of the long-time averaged $U$ velocity profiles at the outlet for grids $\mathrm{a}, \mathrm{b}$ and $\mathrm{c}$.

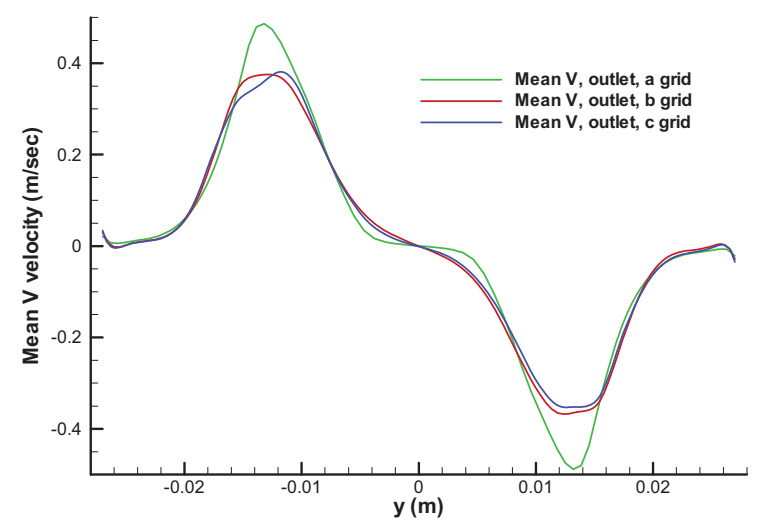

Figure 10. Comparison of the long-time averaged V velocity profiles at the outlet for grids $\mathrm{a}, \mathrm{b}$ and $\mathrm{c}$.

The profiles shown in Figs. 9 and 10 (whose nodes are not coincident) can also be compared mathematically using an $L_{2}$ norm based on a finite-element-style integration over the domain of the profile. The $L_{2}$ norm is computed based on the formula:

$$
L_{2}=\frac{\left\{\int_{2}\left[s(y, t)-s_{o}\left(y, t_{o}\right)\right]^{2} d y\right\}^{1 / 2}}{\left\{\int_{2} s_{o}^{2}\left(y, t_{o}\right) d y\right\}^{1 / 2}}
$$

where $\Omega$ is the domain of the profile. Using Eqn. 2, with the finest grid ' $c$ ' being used as the reference profile, the $L_{2}$ norm for profiles for the ' $a$ ' and ' $b$ ' grids are $6.1 \%$ and $2.1 \%$ for the $\mathrm{U}$ velocity and $22.2 \%$ and $5.7 \%$ for the $\mathrm{V}$ velocity.

With a provisional grid chosen, additional simulations can be made to compare velocity profiles for the two different outlet boundary conditions for the 'inside-only' CFD models with the 'inner+outer' CFD model. The inside geometry for all of these simulations is given in Fig. 3.

Initial computations were made using the CFD model that includes the outer flow domain. Figure 11 illustrates the stream function [3] at a point in time of the flow. As can be seen, there is vortex-shedding occurring behind the thick walls of the physical model that interacts with the flow exiting from the model, which flow also contains shedding vortices. Based on this observation alone, one can conclude that, at least at the exit plane of the physical model, the flow simulations for the inside-only CFD models cannot be the same as those for the CFD model that includes the outer flow. Also, vortices are being shed behind every full and half cylinder.

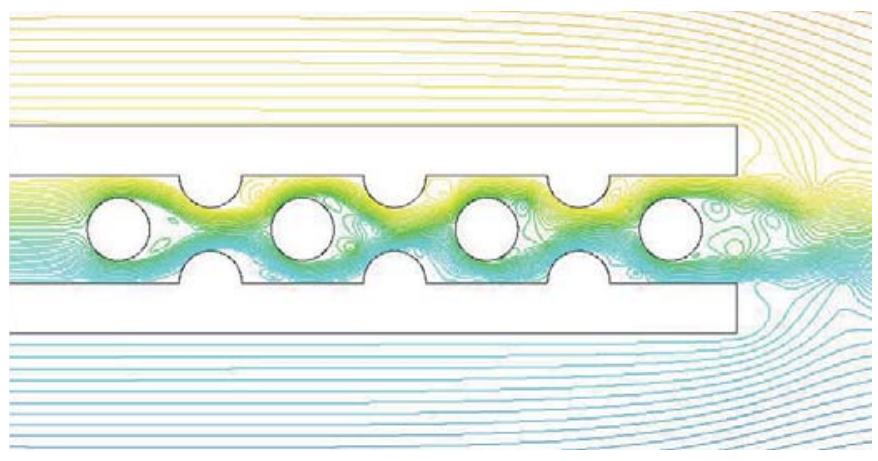

Figure 11. Stream function contours of the flow in the CFD model that includes the outer flow showing vortex-shedding behind the physical model in the MIR test loop.

Long-time calculations were made for the three CFD models, namely, the 'inside-only' models using the 'pressureoutlet' and the 'outflow' boundary conditions and the 'inner+outer' model using $\Delta \mathrm{t}=7.5 \times 10^{-5}$ secs. To help see if the simulations have been made for a sufficiently long time period, the $l_{2}$ norm of the outlet plane profiles for several points in time has been computed for each model as was done previously using Eqn. 1 . Figures $12-14$ plot the $l_{2}$ norm results as functions of time for the 3 cases.

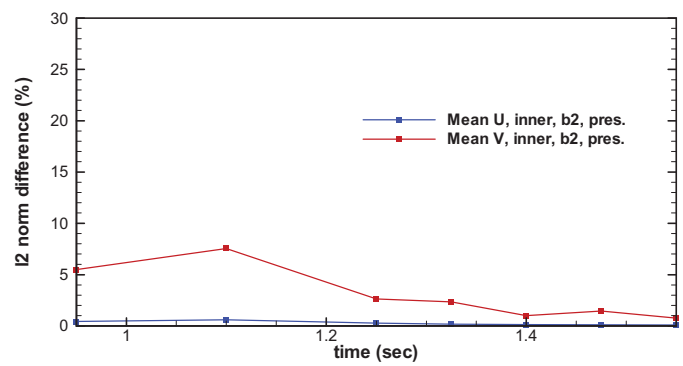

Figure 12. $l_{2}$ differences for the 'inside-only' 'pressure-outlet' model. Ref. time $=1.5932 \mathrm{sec}$. 


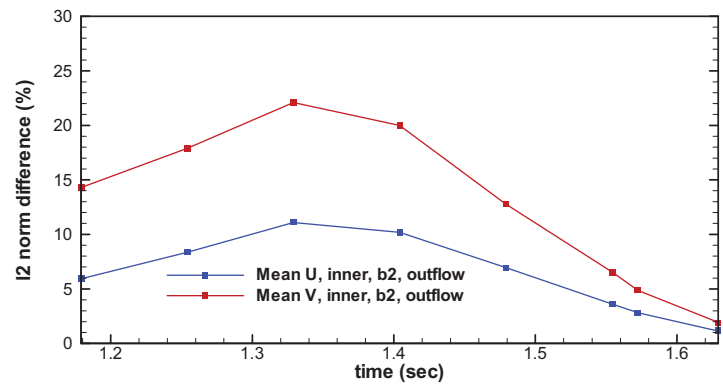

Figure 13. $l_{2}$ differences for the 'inside-only' 'outflow' model. Ref. time $=1.6953 \mathrm{sec}$.

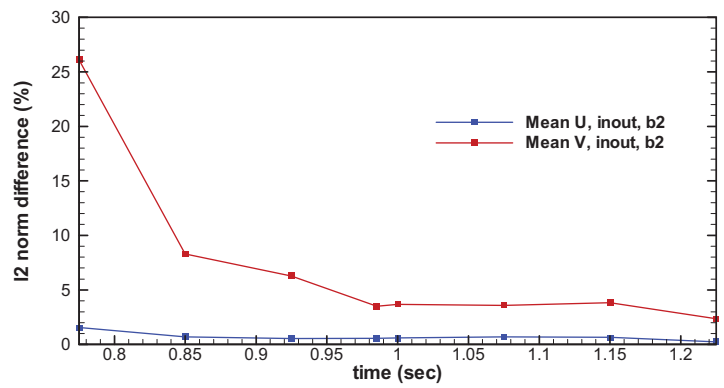

Figure 14. $l_{2}$ differences for the 'inner+outer' model. Ref. time $=1.2909 \mathrm{sec}$.

The time profiles of the $l_{2}$ norms for the 3 cases appear to show adequate time for the long-time averages though additional time could be added to ensure such.

Additional information can be obtained from plotting time signature data from points in the flow fields. Figure 15 illustrates the locations of points from which data are plotted. Figure 16 plots the long-time-averaged velocities $U$ at points p3 and p4 and the Reynolds-averaged velocity $u$ at point p5 for the 'inner+outer' model. The mean velocities $\mathrm{U}$ at $\mathrm{p} 3$ and p4 appear to be stationary at this scale. The Reynoldsaveraged velocity $u$ at $p 5$ exhibits at least two time scales of unsteadiness. The time scale of the smallest cycle ranges from $0.01-0.02$ secs, while a pattern that repeats itself over 11 of the smaller cycles is apparent; the time scale of the larger pattern is 0.1634 secs. $(1.0573-1.220725$ sec.) Figure 17 plots the two mean velocities of Fig. 16 at an expanded scale; it appears that the long-time averages are still changing in time. Additional simulations could be made for longer times to ensure that long-time averages are changing negligibly.

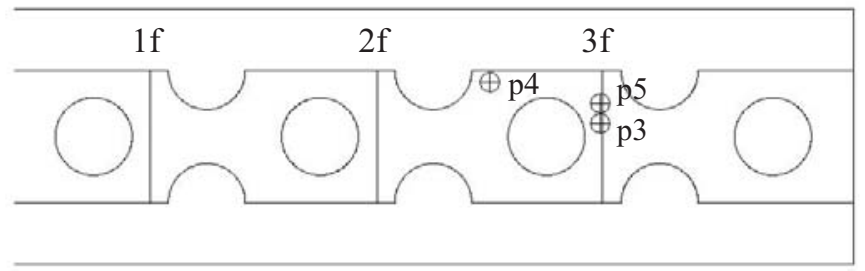

Figure 15. Locations of points and profile lines. Flow is left to right.

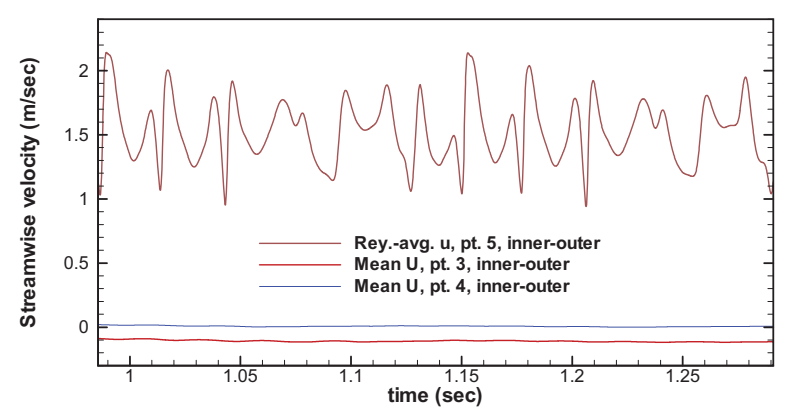

Figure 16. Long-time-averaged velocities $U$ at points $\mathrm{p} 3$ and p4 and Reynolds-averaged velocity $u$ at point p5; see Fig. 15 for point locations.

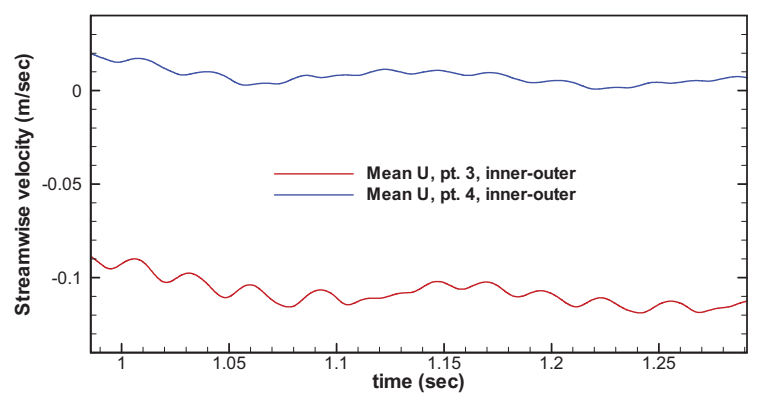

Figure 17. Same data from Fig. 16 at points p3 and p4.

Figures 18 and 19 plot the Reynolds-averaged and longtime-averaged velocities at point $\mathrm{p} 5$ for the 'inside-only' CFD models using the 'pressure-outlet' and 'outflow' conditions, respectively. For the 'pressure-outlet' case, a pattern of 11 cycles with overall length of 0.1638 secs $(1.3898-1.5536$ sec.) can be detected that matches the form of the longer time pattern of the 'inner+outer' case of Fig. 15 for the same p5. The pattern for the 'outflow' case of Fig. 19 does not resemble the patterns of the other two cases. The presence of multiple time scales is likely due to the presence of multiple asynchronous vortex-shedding sites. Note that the time signatures of Figs. 16, 18 and 19 are rather different from that of Fig. 5, where there were fewer vortex-shedding sites (only two full and four half posts compared to four full posts and six half posts).

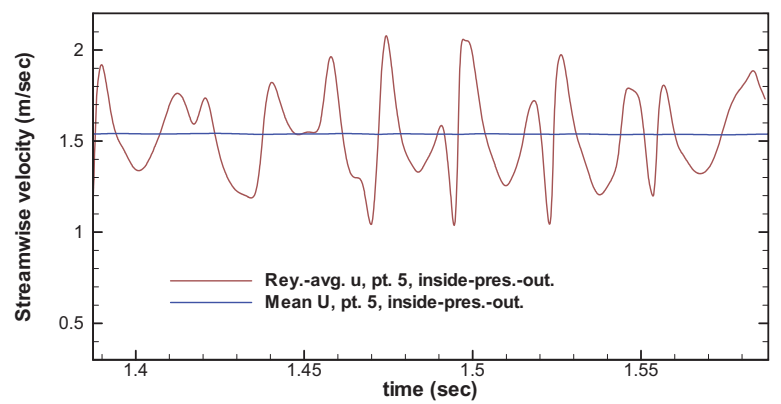

Figure 18. Long-time-averaged and Reynolds-averaged velocity at point $\mathrm{p} 5$ for 'pressure-outlet' case. 


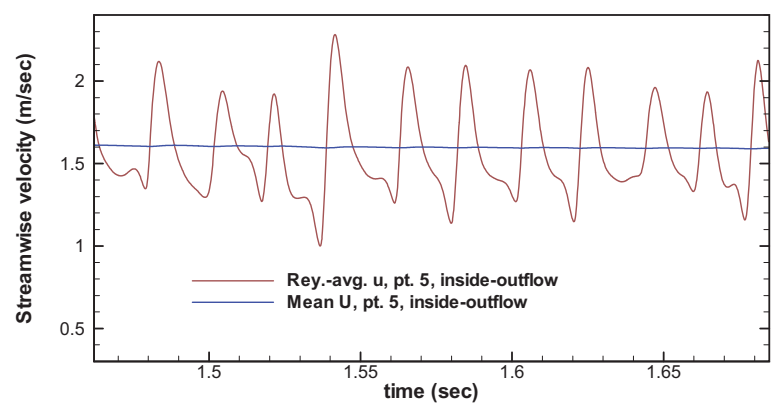

Figure 19. Long-time-averaged and Reynolds-averaged velocity at point $\mathrm{p} 5$ for 'outflow' case.

Figure 20 plots the long- time-averaged velocity $\mathrm{U}$ at point 5 of Fig. 18 for the 'pressure-outlet' case at an expanded scale. Similar to the plots at points 3 and 4 of Fig. 16, the long-time-average velocity appears to be still changing.

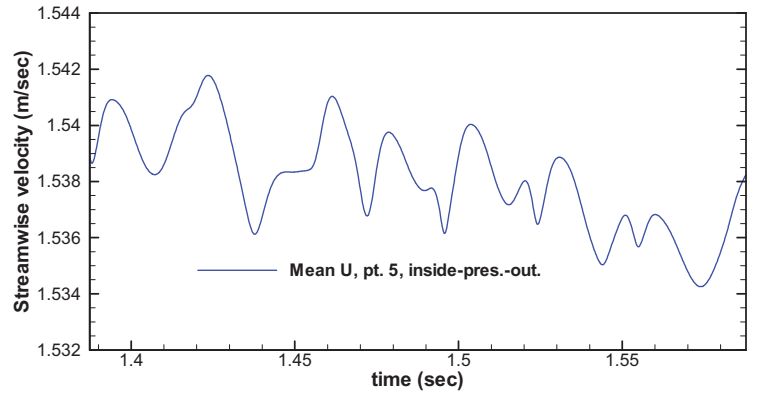

Figure 20. Long-time-averaged velocity $\mathrm{U}$ at point $\mathrm{p} 5$ from Fig. 18 ,

Velocity profiles are now compared for the three CFD models. Figures 21-22 illustrate the long-time averaged U and $\mathrm{V}$ velocities at the physical model outlet, for the cases of the inside-only CFD models using 'pressure-outlet' and 'outflow' boundary conditions and for the 'inner+outer' CFD model. Clearly, neither of the velocity profiles for the 'inside-only' models matches results with the 'inner+outer' model. However, results using the 'pressure-outlet' boundary condition come much closer to the 'inner+outer' results than do results using the 'outflow' boundary condition.

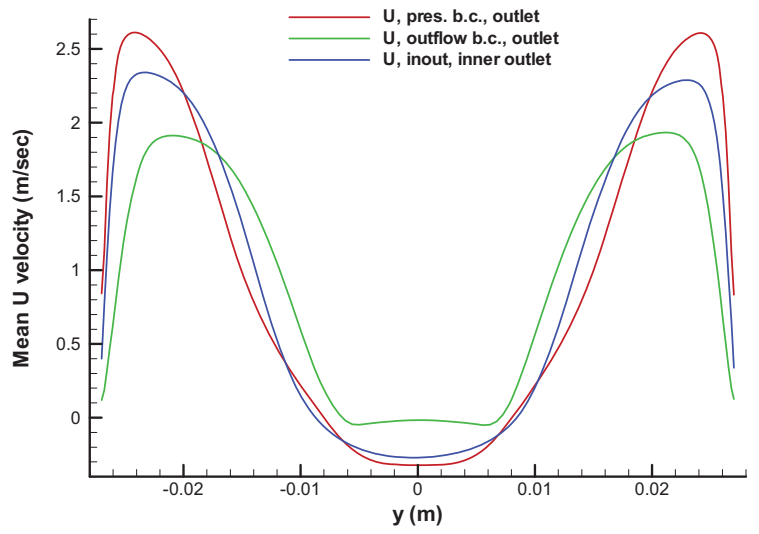

Figure 21. Profiles of long-time averaged U velocity at the physical model outlet for the inside-only CFD models using 'pressure-outlet' and 'outflow' conditions and for the 'inner+outer' CFD model.

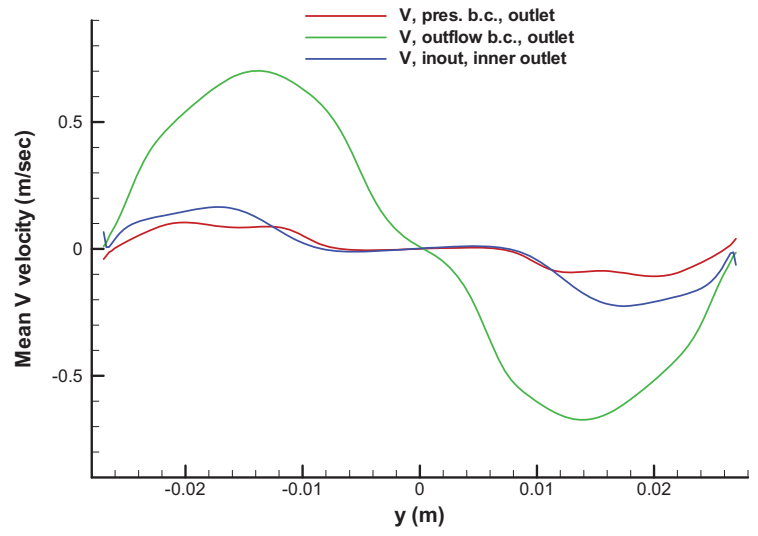

Figure 22. Same as Fig. 21 for long-time averaged V velocity.

Velocity profiles at three additional locations are compared for the three CFD models to see the relative effects of the outlet boundary condition on upstream results and to see if either of the 'inside-only' CFD model results compare well with those for the 'inner+outer' CFD model.

Figures 23-28 illustrate long-time averaged $\mathrm{U}$ and $\mathrm{V}$ velocity profiles at axial locations $1 \mathrm{f}, 2 \mathrm{f}$ and $3 \mathrm{f}$. These locations are shown in Fig. 15. The times of comparisons are at the reference times of Figs. 12-14. Here, the profiles for the 'inside-only' case using the 'pressure-outlet' condition are remarkably close to those for the 'inner+outer' case. Although these results are not considered validated, it is clear that results for the 'inside-only' CFD model using the 'pressure-outlet' boundary condition yields very similar results to the full 'inner+outer' CFD model at least from profile line ' $3 \mathrm{f}$ ' and upstream. This means that the region on the outside of the physical model need not be included in the CFD model. The results for the 'inside-only' case using the 'outflow' condition are noticeably inferior to the 'pressure-outlet' results, though they still show fair agreement with the 'inner+outer' computations.

In addition to the long-time averaged velocities, it is interesting to examine the results for the Reynolds shear stress. Figures 29-31 plot long-time-averaged Reynolds shear stress at locations $1 \mathrm{f}, 2 \mathrm{f}$ and $3 \mathrm{f}$ for the three CFD models. The profiles for the 'inside-only' 'pressure-outlet' case are again remarkably close to those for the 'inner+outer' model. And the results for the 'inside-only' 'outflow' case are still in fair agreement with the full CFD model.

The results for the 'inside-only' 'pressure-outlet' model are from simulation time 1.5932 secs while those for the 'inner+outer' case are from 1.2909 secs. This time difference is about two times the large $0.1638 \mathrm{sec}$ patterns detected in Figs. 16 and 18 . With such a difference in the times of the two cases and in view of the remarkable agreement between their respective results upstream of the outlet, it appears that the long-time averages are not changing significantly and that extending the times of the calculations is not necessary. 


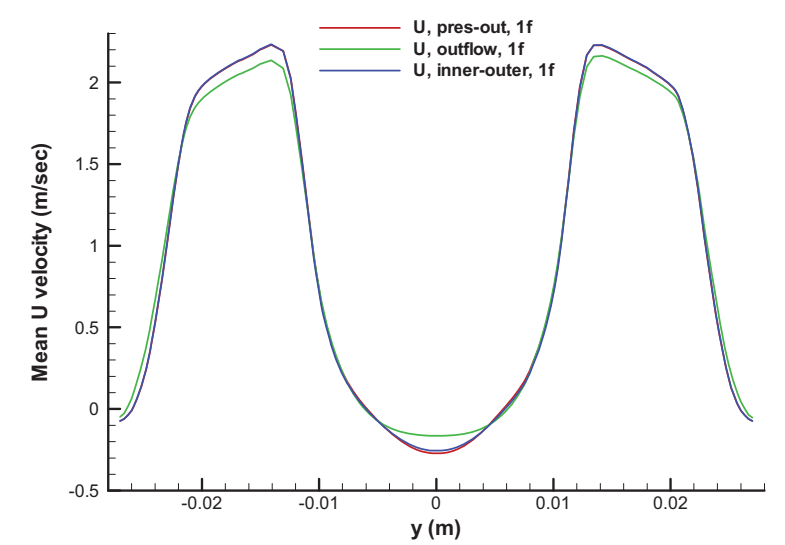

Figure 23. U velocity profiles at location $1 \mathrm{f}$.

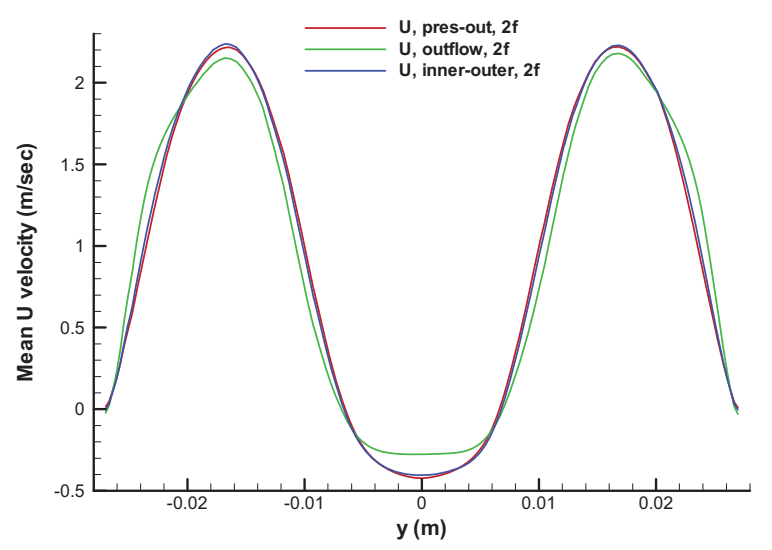

Figure 24 . U velocity profiles at location $2 f$.

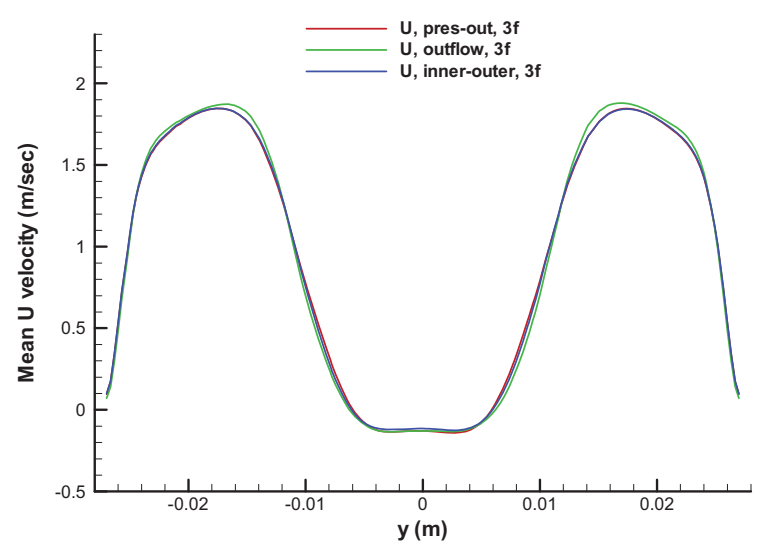

Figure 25. U velocity profiles at location $3 \mathrm{f}$.

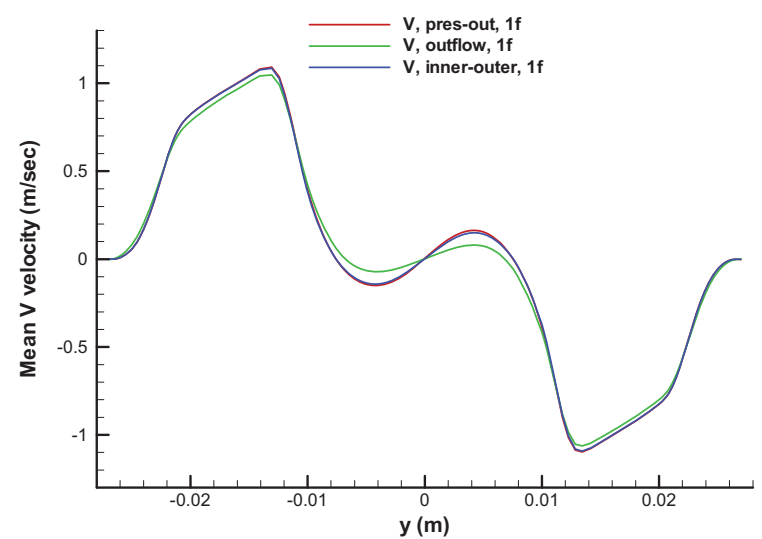

Figure 26. V velocity profiles at location $1 \mathrm{f}$.

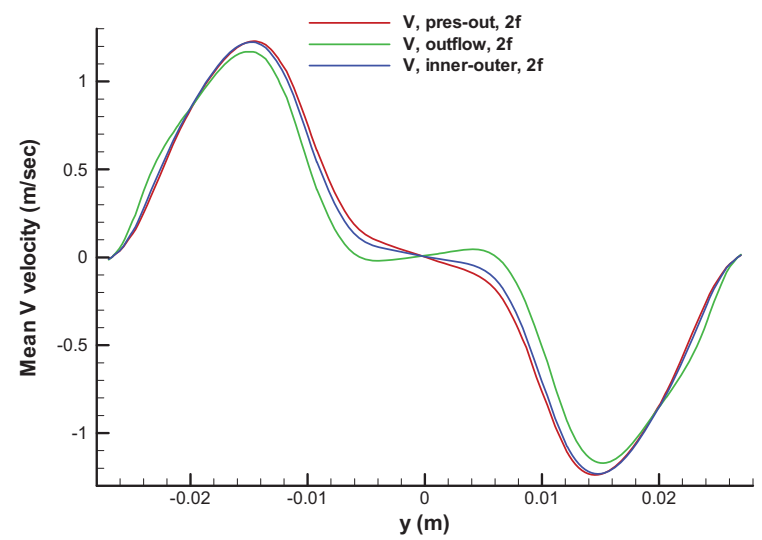

Figure 27 . V velocity profiles at location $2 \mathrm{f}$.

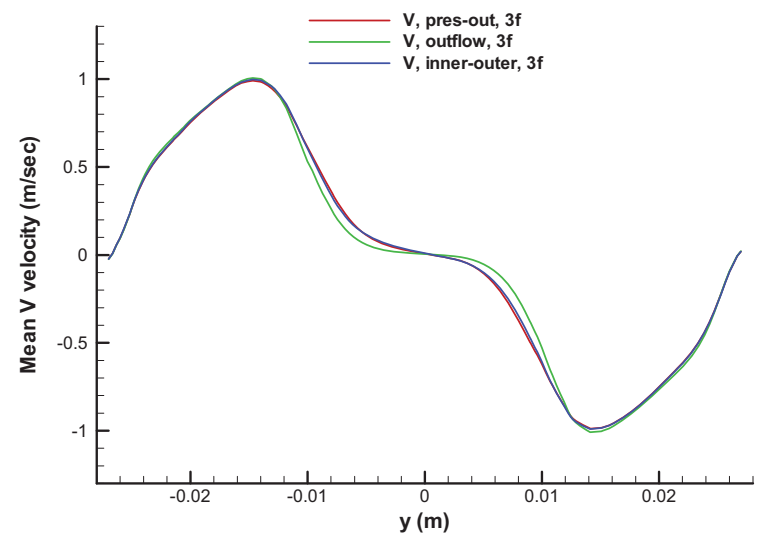

Figure 28 . V velocity profiles at location $3 \mathrm{f}$. 


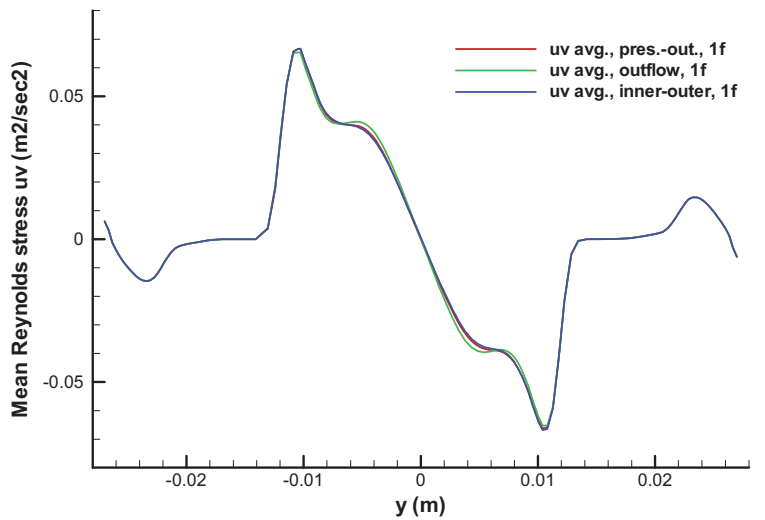

Figure 29. Long-time-averaged Reynolds stress $\overline{u v}$ at location $1 \mathrm{f}$ for the three CFD models.

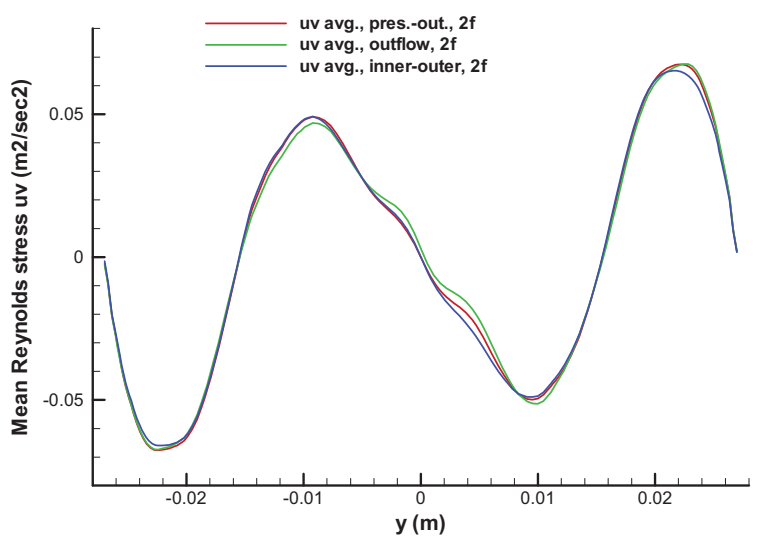

Figure 30. Long-time-averaged Reynolds stress $\overline{u v}$ at location $2 \mathrm{f}$ for the three CFD models.

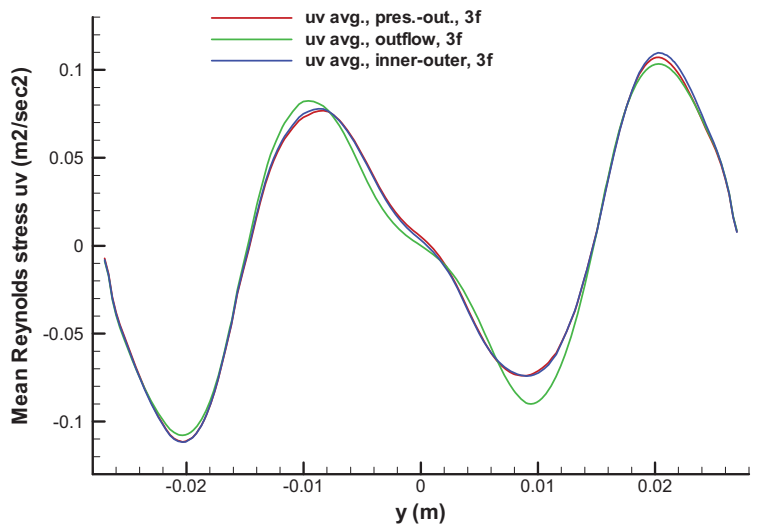

Figure 31. Long-time-averaged Reynolds stress $\overline{u v}$ at location $3 \mathrm{f}$ for the three CFD models.

\section{SUMMARY}

In summary, investigations were made to help devise an analysis plan for the CFD analysis of the three-dimensional scaled model of a portion of a prismatic VHTR lower plenum. The model was built and installed in the INL MIR test facility where experimental data were taken of the three-dimensional flow field using particle image velocimetry (PIV). Employing 2D CFD models of the scaled physical model wherein most of the interior of the model was replicated, it has been found that the inclusion of the outer flow around the model is not necessary to obtain adequate numerical representations of the flow field inside of the model, but upstream of the exit and not including the exit region. The 'pressure-outlet' condition, available in FLUENT, provides adequate simulations when compared to the case where the outer flow is included in the flow domain. The case where the 'outflow' outlet boundary condition is used produces only fair agreement with the 'inner+outer' calculations. The former condition applies a constant static pressure just beyond the exit plane of the outlet; the latter enforces constant derivatives for the velocities at the exit plane.

Furthermore, it was found that for the 2D models, at least, the realizable $\mathrm{k} \sim \varepsilon$ model using either the enhanced wall model or standard wall functions in FLUENT [3] with a grid suitable for the enhanced wall model does not predict vortex shedding. The second moment Reynolds stress model (RSM) using standard wall functions does provide for vortex shedding even without a 'numerical kick'.

\section{ACKNOWLEDGEMENTS}

This manuscript has been authored by Battelle Energy Alliance, LLC under Contract No. DE-AC07-05ID14517 with the U.S. Department of Energy. The United States Government retains and the publisher, by accepting the article for publication, acknowledges that the United States Government retains a nonexclusive, paid-up, irrevocable, world-wide license to publish or reproduce the published form of this manuscript, or allow others to do so, for United States Government purposes.

\section{REFERENCES}

[1] General Atomics, 1996. Gas Turbine - Modular Helium Reactor (GT-MHR) Conceptual Design Description Report, Doc. 910720, Rev. 1.

[2] H. M. McIlroy, D. M. McEligot and R. J. Pink, Measurement of Flow Phenomena in a Lower Plenum Model of a Prismatic Gas-Cooled Reactor, Proceedings of the $16^{\text {th }}$ International Conference on Nuclear Engineering (ICONE-16), Orlando, May 11-15, 2008.

[3] FLUENT, version 6.3.26, 2008. FLUENT Inc., 10 Cavendish Court, Centerra Resource Park, Lebanon, NH, 03766.

[4] R. W. Johnson, "Modeling Strategies for Unsteady Turbulent Flows in the Lower Plenum of the VHTR," Nuclear Engineering \& Design, 238, 482-491, 2008.

[5] H. M. McIlroy, VHTR Lower Plenum 3D PIV Study, INL Laboratory Notebook LAB-184, pp. 105-148 of book 1 and 5-34 of book 2 . 